\title{
Weak Solutions for a Sixth Order Cahn-Hilliard Type Equation with Degenerate Mobility
}

\author{
Aibo Liu and Changchun Liu \\ Department of Mathematics, Jilin University, Changchun 130012, China \\ Correspondence should be addressed to Changchun Liu; liucc@jlu.edu.cn \\ Received 29 January 2014; Accepted 4 March 2014; Published 1 April 2014 \\ Academic Editor: Ming Mei
}

Copyright ( 2014 A. Liu and C. Liu. This is an open access article distributed under the Creative Commons Attribution License, which permits unrestricted use, distribution, and reproduction in any medium, provided the original work is properly cited.

We study an initial-boundary problem for a sixth order Cahn-Hilliard type equation, which arises in oil-water-surfactant mixtures. An existence result for the problem with a concentration dependent diffusional mobility in three space dimensions is presented.

\section{Introduction}

We consider

$$
\begin{gathered}
u_{t}-\operatorname{div}\left[m ( u ) \left(k \nabla \Delta^{2} u+\nabla\left(-a(u) \Delta u-\frac{a^{\prime}(u)}{2}|\nabla u|^{2}\right.\right.\right. \\
+f(u)))]=0,
\end{gathered}
$$

in $\Omega \times(0, T)$, where $\Omega \subset \mathbb{R}^{3}$ is a bounded domain, $m(u)=|u|^{n}$, $n>1$ is mobility, $k>0, a(u)=a_{1} u^{2}+a_{2}$, and $a_{1}>0, a_{2}$ are constants [1]. From the physical consideration, we prefer to consider a typical case of the volumetric free energy $F(u)$; that is, $F^{\prime}(u)=f(u)$, in the following form $[1,2]$ :

$$
F(u)=(u+1)^{2}\left(u^{2}+h_{0}\right)(u-1)^{2} .
$$

Equation (1) is supplemented by the boundary value conditions

$$
\left.u\right|_{\partial \Omega}=\left.\Delta u\right|_{\partial \Omega}=\left.\Delta^{2} u\right|_{\partial \Omega}=0, \quad t>0
$$

and the initial value condition

$$
u(x, 0)=u_{0}(x) .
$$

Equation (1) is the sixth order parabolic equation which describes dynamics of phase transitions in ternary oil-watersurfactant systems [2]. Here $u(x, t)$ is the scalar order parameter which is proportional to the local difference between oil and water concentrations. Pawłow and Zajączkowski [2] proved that the initial-boundary value problem (1)-(4) with $m(u)=1$ admits a unique global smooth solution which depends continuously on the initial datum. Wang and Liu [3] proved that the solutions of problem (1)-(4) with $m(u)=$ 1 might not be classical globally. In other words, in some cases, the classical solutions exist globally, while in some other cases, such solutions blow up at a finite time. They also discussed the existence of global attractor. Liu and Wang [4] considered the optimal control problem for the problem (1)(4) with $m(u)=1$. They proved the existence of optimal solution. The optimality system is also established. Since the mobility depends on the concentration in general, the equation with nonlinear main part reflects even more exactly the physical reality comparing to the one with linear main part. Schimperna and Pawłow [5] studied (1) with viscous term $\Delta u_{t}, m(u)=1$ and logarithmic potential

$$
\begin{array}{r}
F(r)=(1-r) \log (1-r)+(1+r) \log (1+r)-\frac{\lambda}{2} r^{2}, \\
\lambda>0 .
\end{array}
$$

They investigated the behavior of the solutions to the sixth order system as the parameter $\gamma$ tends to 0 . The uniqueness and regularization properties of the solutions have been discussed. Liu [6] studied the problem (1)-(4) and he proved the existence of classical solutions for two dimensions and $m(s) \in C^{1+\alpha}(\mathbb{R}), M_{1} \leq m(s),\left|m^{\prime}(s)\right|^{2} \leq M_{2} m(s)$. 
In this paper, we study the problem (1)-(4) with degenerate concentration dependent mobility. The main difficulties for treating the problem are caused by the degeneracy of the principal part, nonlinearity of the fourth order term, and the lack of maximum principle. Our method is based on Galerkin approximation and Simon's compactness results.

This paper is organized as follows. In Section 2, using Galerkin approximation, we prove the existence of the weak solution for positive mobility. In Section 3, we prove the existence of the weak solution for degenerate case.

\section{Existence for Positive Mobilities}

In this section, we study the Cahn-Hilliard equation with a mobility which is bounded away from zero. We prove existence of weak solutions. tion:

Consider the following sixth order Cahn-Hilliard equa-

$$
\begin{aligned}
& u_{t}=\operatorname{div}(m(u) \nabla \omega), \\
& \omega=k \Delta^{2} u-a(u) \Delta u-\frac{a^{\prime}(u)}{2}|\nabla u|^{2}+f(u),
\end{aligned}
$$

with the boundary conditions

$$
\left.u\right|_{\partial \Omega}=\left.\Delta u\right|_{\partial \Omega}=\left.\Delta^{2} u\right|_{\partial \Omega}=0, \quad t>0
$$

and the initial value

$$
u(x, 0)=u_{0}(x) .
$$

In this section, we assume $\Omega$ is a bounded domain with Lipschitz boundary and $m$ such that $m(s)$.

(i) $m(s) \in C\left(R, R^{+}\right)$and there is $m_{1}>0$ such that $m_{1} \leq$

Under these assumptions we can state the following theorem.

Theorem 1. Suppose $u_{0} \in H^{2}(\Omega)$. Then there exists a pair of functions $(u, \omega)$ such that

(1) $u \in L^{\infty}\left(0, T ; H^{2}(\Omega)\right) \cap C\left([0, T] ; L^{2}(\Omega)\right)$,

(2) $u_{t} \in L^{2}\left(0, T ;\left(H^{1}(\Omega)\right)^{\prime}\right)$,

(3) $u(0)=u_{0}$,

(4) $\omega \in L^{2}\left(0, T ; H^{1}(\Omega)\right)$,

which satisfies equation (1) in the following weak sense:

$$
\int_{0}^{T}\left\langle\xi(t), u_{t}(t)\right\rangle_{H^{1},\left(H^{1}\right)^{\prime}} d t=-\int_{\Omega_{T}} m(u) \nabla \omega \nabla \xi d x d t,
$$

for all $\xi \in L^{2}\left(0, T ; H^{1}(\Omega)\right)$, and

$$
\begin{aligned}
\int_{\Omega} \omega \phi d x= & k \int_{\Omega} \Delta u \Delta \phi d x \\
& +\int_{\Omega}\left(-a(u) \Delta u-\frac{a^{\prime}(u)}{2}|\nabla u|^{2}+f(u)\right) \phi d x
\end{aligned}
$$

for all $\phi \in H^{2}(\Omega)$, and almost all $t \in[0, T]$.
Proof. To prove the theorem we apply a Galerkin approximation. Let $\left\{\phi_{i}\right\}_{i \in N}$ be the eigenfunctions of the Laplace operator with Neumann boundary conditions; that is,

$$
\begin{aligned}
& -\Delta \phi_{i}=\lambda_{i} \phi_{i}, \quad \text { in } \Omega, \\
& \nabla \phi_{i} \cdot n=0, \quad \text { on } \partial \Omega .
\end{aligned}
$$

The eigenfunction $\phi_{i}$ is orthogonal in the $L^{2}(\Omega), H^{1}(\Omega)$, and $H^{2}(\Omega)$ scalar product. We normalize the $\phi_{i}$ such that $\left(\phi_{i}, \phi_{j}\right)_{L^{2}(\Omega)}=\delta_{i j}$. Furthermore we assume without loss of generality that $\lambda_{1}=0$. Now we consider the following Galerkin ansatz for (6):

$$
u^{N}(t, x)=\sum_{i=1}^{N} c_{i}^{N}(t) \phi_{i}(x), \quad \omega^{N}(t, x)=\sum_{i=1}^{N} d_{i}^{N}(t) \phi_{i}(x),
$$

$\int_{\Omega} \partial_{t} u^{N} \phi_{j} d x=-\int_{\Omega} m\left(u^{N}\right) \nabla \omega^{N} \nabla \phi_{j} d x, \quad j=1,2, \ldots, N$,

$$
\begin{gathered}
\int_{\Omega} \omega^{N} \phi_{j} d x \\
=k \int_{\Omega} \Delta u^{N} \Delta \phi_{j} d x \\
+\int_{\Omega}\left(-a\left(u^{N}\right) \Delta u^{N}\right. \\
\left.-\frac{a^{\prime}\left(u^{N}\right)}{2}\left|\nabla u^{N}\right|^{2}+f\left(u^{N}\right)\right) \phi_{j} d x, \\
u^{N}(0)=\sum_{i=1}^{N}\left(u_{0}, \phi_{i}\right)_{L^{2}(\Omega)} \phi_{i} .
\end{gathered}
$$

This gives an initial value problem for a system of ordinary differential equations for $\left(c_{1}, c_{2}, \ldots, c_{N}\right)$ :

$$
\partial_{t} c_{j}^{N}=-\sum_{k=1}^{N} d_{k}^{N} \int_{\Omega} m\left(\sum_{i=1}^{N} c_{i}^{N} \phi_{i}\right) \nabla \phi_{k} \nabla \phi_{j} d x
$$

$$
\begin{aligned}
& d_{j}^{N} \\
& =k \lambda_{j}^{2} c_{j}^{N}+\int_{\Omega} a\left(\sum_{i=1}^{N} c_{i}^{N} \phi_{i}\right) \lambda_{j} c_{j}^{N} d x \\
& +\int_{\Omega}\left(-\frac{a^{\prime}\left(\sum_{i=1}^{N} c_{i}^{N} \phi_{i}\right)}{2}\left|\sum_{i=1}^{N} c_{i}^{N} \nabla \phi_{i}\right|^{2}+f\left(\sum_{i=1}^{N} c_{i}^{N} \phi_{i}\right)\right) \\
& \times \phi_{j} d x, \\
& c_{j}^{N}(0)=\left(u_{0}, \phi_{j}\right)_{L^{2}(\Omega)} .
\end{aligned}
$$


By use of the Peano existence theorem, the initial value problem has a local solution. Now, we set

$$
E(t)=\int_{\Omega}\left(\frac{k}{2}\left(\Delta u^{N}\right)^{2}+\frac{a\left(u^{N}\right)}{2}\left|\nabla u^{N}\right|^{2}+F\left(u^{N}\right)\right) d x
$$

In order to derive a priori estimates we differentiate the $E(t)$ and get

$$
\begin{array}{r}
\frac{d}{d t} E(t)=\int_{\Omega}\left(k \Delta u^{N} \Delta u_{t}^{N}+\frac{a^{\prime}\left(u^{N}\right)}{2}\left|\nabla u^{N}\right|^{2} u_{t}^{N}\right. \\
\left.+a\left(u^{N}\right) \nabla u^{N} \nabla u_{t}^{N}+f\left(u^{N}\right) u_{t}^{N}\right) d x \\
=\int_{\Omega}\left(k \Delta u^{N} \Delta u_{t}^{N}-\frac{a^{\prime}\left(u^{N}\right)}{2}\left|\nabla u^{N}\right|^{2} u_{t}^{N}\right. \\
\left.\quad-a\left(u^{N}\right) \Delta u^{N} u_{t}^{N}+f\left(u^{N}\right) u_{t}^{N}\right) d x \\
=\int_{\Omega} \omega^{N} u_{t}^{N} d x=-\int_{\Omega} m\left(u^{N}\right)\left|\nabla \omega^{N}\right|^{2} d x .
\end{array}
$$

This implies

$$
\begin{gathered}
\int_{\Omega}\left(\frac{k}{2}\left(\Delta u^{N}\right)^{2}+\frac{a\left(u^{N}\right)}{2}\left|\nabla u^{N}\right|^{2}+F\left(u^{N}\right)\right) d x \\
+\int_{\Omega_{T}} m\left(u^{N}\right)\left|\nabla \omega^{N}\right|^{2} d x d t \\
=\int_{\Omega}\left(\frac{k}{2}\left(\Delta u^{N}(0)\right)^{2}+\frac{a\left(u^{N}(0)\right)}{2}\left|\nabla u^{N}(0)\right|^{2}\right. \\
\left.+F\left(u^{N}(0)\right)\right) d x \leq C .
\end{gathered}
$$

The last inequality follows the fact that $u_{0} \in H^{2}(\Omega)$.

On the other hand, we have

$$
\int_{\Omega}\left|\nabla u^{N}(x, t)\right|^{2} d x \leq \varepsilon \int_{\Omega}\left(\Delta u^{N}\right)^{2} d x+C(\varepsilon) \int_{\Omega}\left(u^{N}\right)^{2} d x .
$$

By the Young inequality

$$
\left(u^{N}\right)^{2} \leq \varepsilon\left(u^{N}\right)^{6}+C_{1 \varepsilon}, \quad\left(u^{N}\right)^{4} \leq \varepsilon\left(u^{N}\right)^{6}+C_{2 \varepsilon} .
$$

Combining the above inequalities and using $a(u)=a_{1} u^{2}+a_{2}$, $a_{1}>0$ yield

$$
\text { ess } \sup _{0<t<T}\|u(t)\|_{H^{2}(\Omega)} \leq C \text {. }
$$

This estimate implies that the $\left(C_{1}^{N}, \ldots, C_{N}^{N}\right)$ are bounded and therefore a global solution to the initial value problem (6) exists. By Gagliardo-Nirenberg inequality (noticing that we consider only the three-dimensional case)

$$
\sup _{\Omega}|u| \leq C_{1}\|\Delta u\|_{L^{2}}^{3 / 4}\|u\|_{L^{2}}^{1 / 4} \leq C .
$$

If we denote by $\Pi_{N}$ the projection of $L^{2}(\Omega)$ onto span $\phi_{1}, \ldots, \phi_{N}$, we get

$$
\begin{aligned}
\left|\int_{\Omega_{T}} \partial_{t} u^{N} \phi d x d t\right|= & \left|\int_{\Omega_{T}} \partial_{t} u^{N} \Pi_{N} \phi d x d t\right| \\
= & \left|\int_{\Omega_{T}} m\left(u^{N}\right) \nabla \omega^{N} \nabla \Pi_{N} \phi d x d t\right| \\
\leq & \left(\int_{\Omega_{T}}\left|m\left(u^{N}\right) \nabla \omega^{N}\right|^{2} d x d t\right)^{1 / 2} \\
& \times\left(\int_{\Omega_{T}}\left|\nabla \Pi_{N} \phi\right|^{2} d x d t\right)^{1 / 2} \\
\leq & M\left(\int_{\Omega_{T}} m\left(u^{N}\right)\left|\nabla \omega^{N}\right|^{2} d x d t\right)^{1 / 2} \\
& \times\|\nabla \phi\|_{L^{2}\left(\Omega_{T}\right)} \leq C\|\nabla \phi\|_{L^{2}\left(\Omega_{T}\right)}
\end{aligned}
$$

for all $\phi \in L^{2}\left(0, T ; H^{1}(\Omega)\right)$. This implies

$$
\left\|\partial_{t} u^{N}\right\|_{L^{2}\left(0, T ;\left(\left(H^{1}(\Omega)\right)^{\prime}\right)\right.} \leq C .
$$

Using compactness results, (Simon [7] and Lions [8]) we obtain for a subsequence (which we still denote by $u^{N}$ )

$$
\begin{gathered}
u^{N} \longrightarrow u, \quad \text { weak }^{*} \text { in } L^{\infty}\left(0, T ; H^{2}(\Omega)\right), \\
u^{N} \longrightarrow u, \quad \text { strongly in } C\left(\left[0, T ; L^{2}(\Omega)\right]\right), \\
\partial_{t} u^{N} \longrightarrow u, \quad \text { weakly in } L^{2}\left(0, T ;\left(H^{1}(\Omega)\right)^{\prime}\right), \\
u^{N} \longrightarrow u, \quad \text { strongly in } L^{2}\left(0, T ; L^{\infty}(\Omega)\right),
\end{gathered}
$$

a.e. in $\Omega_{T}$.

It remains to show the convergence of $\omega^{N}$; choosing $j=1$ in (14) gives

$$
\begin{aligned}
& \int_{\Omega} \omega^{N} d x \\
& \quad=\int_{\Omega}\left(-a\left(u^{N}\right) \Delta u^{N}-\frac{a^{\prime}\left(u^{N}\right)}{2}\left|\nabla u^{N}\right|^{2}+f\left(u^{N}\right)\right) d x .
\end{aligned}
$$

By (19) and Poincaré inequality, we obtain

$$
\left\|\omega^{N}\right\|_{L^{2}\left(0, T ; H^{1}(\Omega)\right)} \leq C,
$$

and this implies $\omega^{N} \rightarrow \omega$ weakly in $L^{2}\left(0, T ; H^{1}(\Omega)\right)$. 
The strong convergence of $u^{N}$ in $C\left(\left[0, T ; L^{2}(\Omega)\right]\right)$ and the fact that $u^{N}(0) \rightarrow u_{0}$ in $L^{2}(\Omega)$ give $u(0)=u_{0}$. By $\omega^{N} \rightarrow \omega$ weakly in $L^{2}\left(0, T ; H^{1}(\Omega)\right)$ and $\partial_{t} u^{N} \rightarrow u$ weakly in $L^{2}\left(0, T ;\left(H^{1}(\Omega)\right)^{\prime}\right)$ we can pass limit in (9). Using (22), we know $\left(-a\left(u^{N}\right) \Delta u^{N}-\left(a^{\prime}\left(u^{N}\right) / 2\right)\left|\nabla u^{N}\right|^{2}+h\left(u^{N}\right)\right) \in L^{2}(\Omega)$. Then we can pass the limit in (10).

\section{Existence for the Degenerate Case}

Our approach is to approximate the degenerate problem by nondegenerate equations. By Theorem 1 , we know the existence of weak solution to the problem

$$
\begin{gathered}
u_{t}=\operatorname{div}\left(m_{\varepsilon}(u) \nabla \omega\right), \quad \text { in } \Omega_{T}, \\
\omega=k \Delta^{2} u-a(u) \Delta u-\frac{a^{\prime}(u)}{2}|\nabla u|^{2}+f(u), \quad \text { in } \Omega_{T}, \\
u=\Delta u=\Delta^{2} u=0, \quad \text { on } \partial \Omega, \\
u(x, 0)=u_{0}(x),
\end{gathered}
$$

where $m_{\varepsilon}(u)=m(u)+\varepsilon$.

We denote the solution by $\left(u_{\varepsilon}, \omega_{\varepsilon}\right)$; from now on we assume either $\partial \Omega \in C^{1,1}$ or $\Omega$ is convex.

Lemma 2. The solution $u_{\varepsilon}$ belongs to the space $L^{2}\left(0, T ; H^{4}(\Omega)\right)$ and $\nabla \Delta^{2} u_{\varepsilon} \in L^{2}\left(\Omega_{T}\right)$.

Proof. Since

$$
\begin{aligned}
\int_{\Omega} \omega_{\varepsilon} \phi d x= & k \int_{\Omega} \Delta u_{\varepsilon} \Delta \phi d x \\
& +\int_{\Omega}\left(-a\left(u_{\varepsilon}\right) \Delta u_{\varepsilon}-\frac{a^{\prime}\left(u_{\varepsilon}\right)}{2}\left|\nabla u_{\varepsilon}\right|^{2}\right. \\
& \left.+f\left(u_{\varepsilon}\right)\right) \phi d x
\end{aligned}
$$

for all $\phi \in H^{2}(\Omega)$, and almost all $t \in[0, T]$, from elliptic regularity theory, we get $u_{\varepsilon} \in L^{2}\left(0, T ; H^{4}(\Omega)\right)$. By $\nabla \omega \in$ $L^{2}\left(\Omega_{T}\right)$, (22) and (23), we have

$$
\begin{gathered}
\iint_{\Omega_{T}}\left|\nabla\left(-a\left(u_{\varepsilon}\right) \Delta u_{\varepsilon}-\frac{a^{\prime}\left(u_{\varepsilon}\right)}{2}\left|\nabla u_{\varepsilon}\right|^{2}+h\left(u_{\varepsilon}\right)\right)\right| d x d t \\
=\iint_{\Omega_{T}} \mid-2 a^{\prime}\left(u_{\varepsilon}\right) \nabla u_{\varepsilon} \Delta u_{\varepsilon}-a\left(u_{\varepsilon}\right) \nabla \Delta u_{\varepsilon}+h^{\prime}\left(u_{\varepsilon}\right) \nabla u_{\varepsilon} \\
\quad-\frac{a^{\prime \prime}\left(u_{\varepsilon}\right)}{2} \nabla u_{\varepsilon}\left|\nabla u_{\varepsilon}\right|^{2} \mid d x d t
\end{gathered}
$$

$$
\begin{aligned}
& \leq C \iint_{\Omega_{T}}\left|\nabla u_{\varepsilon}\right|^{2} d x d t+C \iint_{\Omega_{T}}\left|\nabla u_{\varepsilon}\right|^{2} d x d t \\
& \quad+C \iint_{\Omega_{T}}\left|\nabla \Delta u_{\varepsilon}\right|^{2} d x d t \\
& \quad+C \iint_{\Omega_{T}}\left|\nabla u_{\varepsilon}\right|^{3} d x d t \leq C .
\end{aligned}
$$

Then we get

$$
\nabla\left(-a\left(u_{\varepsilon}\right) \Delta u_{\varepsilon}-\frac{a^{\prime}\left(u_{\varepsilon}\right)}{2}\left|\nabla u_{\varepsilon}\right|^{2}+h\left(u_{\varepsilon}\right)\right) \in L^{2}\left(\Omega_{T}\right),
$$

which implies $\nabla \Delta^{2} u_{\varepsilon} \in L^{2}\left(\Omega_{T}\right)$. Therefore, we get

$$
\begin{aligned}
\int_{0}^{T}\left\langle\xi, \partial_{t} u_{\varepsilon}\right\rangle_{H^{1},\left(H^{1}\right)^{\prime}} d t & \\
=-\int_{\Omega_{T}} m_{\varepsilon}\left(u_{\varepsilon}\right) \nabla( & k \Delta^{2} u_{\varepsilon}-a\left(u_{\varepsilon}\right) \Delta u_{\varepsilon} \\
& \left.-\frac{a^{\prime}\left(u_{\varepsilon}\right)}{2}\left|\nabla u_{\varepsilon}\right|^{2}+h\left(u_{\varepsilon}\right)\right) \nabla \xi d x d t
\end{aligned}
$$

for all $\xi \in L^{2}\left(0, T ; H^{1}(\Omega)\right)$.

In the next step we prove the energy estimates.

Lemma 3. There exists an $\varepsilon_{0}$ such that for all $0<\varepsilon<\varepsilon_{0}$, and the following estimates hold with a constant $C$ independent of $\varepsilon$

$$
\begin{gathered}
\text { (1) } \quad \text { ess } \sup _{0<t<T} \int_{\Omega}\left(\frac{k}{2}\left|\Delta u_{\varepsilon}\right|^{2}+\frac{a\left(u_{\varepsilon}\right)}{2}\left|\Delta u_{\varepsilon}\right|^{2}+F\left(u_{\varepsilon}\right)\right) d x \\
+\int_{\Omega_{T}} m\left(u_{\varepsilon}\right)\left|\nabla \omega_{\varepsilon}\right|^{2} d x d t \leq C, \\
\text { (2) } \quad \int_{\Omega_{T}}\left|J_{\varepsilon}\right|^{2} d x d t \leq C, \quad \text { there } J_{\varepsilon}=m_{\varepsilon}\left(u_{\varepsilon}\right) \nabla \omega_{\varepsilon} .
\end{gathered}
$$

Proof. The $\omega_{\varepsilon}=k \Delta^{2} u_{\varepsilon}+\left(-a\left(u_{\varepsilon}\right) \Delta u_{\varepsilon}-\left(a^{\prime}\left(u_{\varepsilon}\right) / 2\right)\left|\nabla u_{\varepsilon}\right|^{2}+\right.$ $\left.f\left(u_{\varepsilon}\right)\right) \in L^{2}\left(0, T ; H^{1}(\Omega)\right)$ is a valid test function in (33). Therefore, we obtain

$$
\begin{gathered}
\int_{0}^{t}\left\langle k \Delta^{2} u_{\varepsilon}+\left(-a\left(u_{\varepsilon}\right) \Delta u_{\varepsilon}-\frac{a^{\prime}\left(u_{\varepsilon}\right)}{2}\left|\nabla u_{\varepsilon}\right|^{2}\right.\right. \\
\left.\left.+f\left(u_{\varepsilon}\right)\right), \partial_{t} u_{\varepsilon}\right\rangle_{H^{1},\left(H^{1}\right)^{\prime}} d t \\
=-\int_{\Omega_{t}} m_{\varepsilon}\left(u_{\varepsilon}\right) \nabla\left|\omega_{\varepsilon}\right|^{2} d x d t, \quad \text { for } \forall t \in[0, T] .
\end{gathered}
$$


Define functions

$$
u_{\varepsilon h}(t, x):=\frac{1}{h} \int_{t-h}^{t} u_{\varepsilon}(\tau, x) d \tau,
$$

where we set $u_{\varepsilon h}(t, x)=u_{0}(x)$ when $t \leq 0$. It is easily proved that

$$
\begin{gathered}
\Delta^{2} u_{\varepsilon h} \longrightarrow \Delta^{2} u_{\varepsilon}, \quad \text { strongly in } L^{2}\left(0, T ; H^{1}(\Omega)\right), \\
\left(-a\left(u_{\varepsilon h}\right) \Delta u_{\varepsilon h}-\frac{a^{\prime}\left(u_{\varepsilon h}\right)}{2}\left|\nabla u_{\varepsilon h}\right|^{2}+f\left(u_{\varepsilon h}\right)\right) \\
\longrightarrow\left(-a\left(u_{\varepsilon}\right) \Delta u_{\varepsilon}-\frac{a^{\prime}\left(u_{\varepsilon}\right)}{2}\left|\nabla u_{\varepsilon}\right|^{2}+f\left(u_{\varepsilon}\right)\right),
\end{gathered}
$$

strongly in $L^{2}\left(0, T ; H^{1}(\Omega)\right)$,

for at least a subsequence $(h \rightarrow 0)$. Furthermore we can show $\partial_{t} u_{\varepsilon h} \rightarrow \partial_{t} u_{\varepsilon}$ strongly in $L^{2}\left(0, T ;\left(H^{1}(\Omega)\right)^{\prime}\right)$. For any $\xi \in L^{2}\left(0, T ; H^{1}(\Omega)\right)$ we have

$$
\begin{aligned}
& \left|\left\langle\xi, \partial_{t} u_{\varepsilon h}-\partial_{t} u_{\varepsilon}\right\rangle_{L^{2}\left(H^{1}\right), L^{2}\left(\left(H^{1}\right)^{\prime}\right)} d t\right| \\
& \quad=\frac{1}{h}\left|\int_{0}^{T}\left\langle\xi, \int_{t-h}^{t}\left(\partial_{t} u_{\varepsilon}(\tau)-\partial_{t} u_{\varepsilon}(t)\right) d \tau\right\rangle_{H^{1},\left(H^{1}\right)^{\prime}} d t\right| \\
& \quad=\frac{1}{h}\left|\int_{0}^{T}\left\langle\xi, \int_{-h}^{0}\left(\partial_{t} u_{\varepsilon}(t+s)-\partial_{t} u_{\varepsilon}(t)\right) d \tau\right\rangle d t\right| \\
& \quad \leq \frac{1}{h} \int_{-h}^{0}\left|\int_{0}^{T} \int_{\Omega} \nabla \xi \cdot\left(J_{\varepsilon}(t+s)-J_{\varepsilon}(t)\right) d x d t\right| d s \\
& \quad \leq\|\nabla \xi\|_{L^{2}\left(\Omega_{T}\right)} \sup _{-h \leq s \leq 0}\left\|J_{\varepsilon}(\cdot+s)-J_{\varepsilon}(\cdot)\right\|_{L^{2}\left(\Omega_{T}\right)} .
\end{aligned}
$$

Since

$$
\sup _{-h \leq s \leq 0}\left\|J_{\varepsilon}(\cdot+s)-J_{\varepsilon}(\cdot)\right\|_{L^{2}\left(\Omega_{T}\right)} \longrightarrow 0, \quad \text { as } h \longrightarrow 0,
$$

it follows that

$$
\partial_{t} u_{\varepsilon h} \longrightarrow \partial_{t} u_{\varepsilon}, \quad \text { strongly in } L^{2}\left(0, T ;\left(H^{1}\right)^{\prime}\right)
$$

Using $\partial_{t} u_{\varepsilon h} \in L^{2}\left(\Omega_{T}\right)$ we have for almost all $t \in[0, T]$

$$
\begin{gathered}
\int_{0}^{t}\left\langle k \Delta^{2} u_{\varepsilon h}+\left(-a\left(u_{\varepsilon h}\right) \Delta u_{\varepsilon h}-\frac{a^{\prime}\left(u_{\varepsilon h}\right)}{2}\left|\nabla u_{\varepsilon h}\right|^{2}\right.\right. \\
\left.\left.+f\left(u_{\varepsilon h}\right)\right), \partial_{t} u_{\varepsilon h}\right\rangle_{H^{1},\left(H^{1}\right)^{\prime}} d t \\
=\int_{0}^{t} \int_{\Omega}\left(k \Delta^{2} u_{\varepsilon h}+\left(-a\left(u_{\varepsilon h}\right) \Delta u_{\varepsilon h}-\frac{a^{\prime}\left(u_{\varepsilon h}\right)}{2}\left|\nabla u_{\varepsilon h}\right|^{2}\right.\right. \\
=\partial_{t} \int_{0}^{t} \int_{\Omega}\left(\frac{k}{2}\left|\Delta u_{\varepsilon h}\right|^{2}+\frac{a\left(u_{\varepsilon h}\right)}{2}\left|\Delta u_{\varepsilon h}\right|^{2}+F\left(u_{\varepsilon h}\right)\right) d x d t \\
=\int_{\Omega}\left(\frac{k}{2}\left|\Delta u_{\varepsilon h}\right|^{2}(t)+\frac{a\left(u_{\varepsilon h}\right)}{2}\left|\nabla u_{\varepsilon h}\right|^{2}(t)+F\left(u_{\varepsilon h}(t)\right)\right) d x \\
\quad-\int_{\Omega} \frac{k}{2}\left|\Delta u_{0}\right|^{2}+\frac{a\left(u_{0}\right)}{2}|\nabla u(0)|^{2}+F\left(u_{0}\right) d x .
\end{gathered}
$$

Pass to the limit $h \rightarrow 0$ in the equation, where we apply the convergence properties of $u_{\varepsilon h}$. Hence, we get

$$
\begin{gathered}
\int_{\Omega}\left(\frac{k}{2}\left|\Delta u_{\varepsilon}\right|^{2}+\frac{a\left(u_{\varepsilon}\right)}{2}\left|\nabla u_{\varepsilon}\right|^{2}+F\left(u_{\varepsilon}\right)\right) d x \\
+\int_{\Omega_{T}} m\left(u_{\varepsilon}\right)\left|\nabla \omega_{\varepsilon}\right|^{2} d x \leq C,
\end{gathered}
$$

and (2) follows easily from (1), and this finishes the proof of Lemma 3.

Theorem 4. Let either $\partial \Omega \in C^{1,1}$ or $\Omega$ convex and suppose that $u_{0} \in H^{1}(\Omega)$. Then there exists a pair $(u, J)$ such that

$$
\begin{aligned}
& \text { (1) } u \in \quad L^{2}\left(0, T ; H^{4}(\Omega)\right) \cap L^{\infty}\left(0, T ; H^{2}(\Omega)\right) \cap \\
& C\left([0, T] ; L^{2}(\Omega)\right), \\
& \text { (2) } u_{t} \in L^{2}\left(0, T ;\left(H^{1}(\Omega)\right)^{\prime}\right), \\
& \text { (3) } u(0)=u_{0} \nabla u \cdot n=0 \text { on } \partial \Omega \times(0, T), \\
& \text { (4) } J \in L^{2}\left(\Omega_{T}, R\right),
\end{aligned}
$$

which satisfies $u_{t}=-\nabla \cdot J$ in $L^{2}\left(0, T ;\left(H^{1}(\Omega)\right)^{\prime}\right)$; that is,

$$
\int_{0}^{T}\left\langle\xi(t), u_{t}(t)\right\rangle_{H^{1},\left(H^{1}\right)^{\prime}} d t=\int_{\Omega_{T}} J \cdot \nabla \xi d x d t
$$

for all $\xi \in L^{2}\left(0, T ; H^{1}(\Omega)\right)$ and

$$
\begin{aligned}
J= & -m(u) \nabla \\
& \cdot\left(k \Delta^{2}(u)+\left(-a(u) \Delta u-\frac{a^{\prime}(u)}{2}|\nabla u|^{2}+f(u)\right)\right),
\end{aligned}
$$


in the following weak sense:

$$
\begin{aligned}
\int_{\Omega_{T}} J \cdot \eta & \\
=\int_{\Omega_{T}} & {\left[k \Delta^{2} u \nabla \cdot(m(u) \eta)-m(u) \nabla\right.} \\
& \left.\times\left(-a(u) \Delta u-\frac{a^{\prime}(u)}{2}|\nabla u|^{2}+f(u)\right) \cdot \eta\right],
\end{aligned}
$$

for all $\eta \in L^{2}\left(0, T ; H^{1}\left(\Omega, R^{3}\right)\right) \cap L^{\infty}\left(\Omega_{T}, R^{3}\right)$ which fulfill $\eta \cdot n=$ 0 on $\partial \Omega \times(0, T)$.

Proof. From Lemma 2, we get

$$
\left\|u_{\varepsilon}\right\|_{L^{2}\left(0, T ; H^{4}(\Omega)\right)} \leq C .
$$

Now we apply the compactness result [7] with $X=H^{4}(\Omega)$, $Y=H^{1}(\Omega)\left(Y=H^{2}(\Omega), H^{3}(\Omega)\right)$, and $Z=\left(H^{1}(\Omega)\right)^{\prime}$ to conclude the existence of a subsequence of $\left(u_{\varepsilon}\right)_{\varepsilon}$ such that

$$
\begin{gathered}
u_{\varepsilon} \longrightarrow u, \quad \text { strongly in } L^{2}\left(\Omega_{T}\right), \\
\nabla u_{\varepsilon} \longrightarrow \nabla u, \quad \text { strongly in } L^{2}\left(\Omega_{T}\right), \\
\nabla \Delta u_{\varepsilon} \longrightarrow \nabla \Delta u, \quad \text { strongly in } L^{2}\left(\Omega_{T}\right), \\
D^{2} u_{\varepsilon} \longrightarrow D^{2} u, \quad \text { strongly in } L^{2}\left(\Omega_{T}\right) .
\end{gathered}
$$

Furthermore using standard compactness properties we obtain the convergence

$$
\begin{aligned}
\partial_{t} u_{\varepsilon} & \longrightarrow \partial_{t} u \quad \text { weakly in } L^{2}\left(0, T ;\left(H^{1}(\Omega)\right)^{\prime}\right), \\
\Delta^{2} u_{\varepsilon} & \longrightarrow \Delta^{2} u \quad \text { weakly in } L^{2}\left(\Omega_{T}\right), \\
J_{\varepsilon} & \longrightarrow J \quad \text { weakly in } L^{2}\left(\Omega_{T}\right) .
\end{aligned}
$$

It remains to show that $u$ fulfills the limit equation. The weak convergence of $\partial_{t} u_{\varepsilon}$ and $J_{\varepsilon}$ gives in the limit

$$
\int_{0}^{T}\left\langle\xi(t), u_{t}(t)\right\rangle_{H^{1},\left(H^{1}\right)^{\prime}} d t=\int_{\Omega_{T}} J \cdot \nabla \xi d x d t
$$

for all $\xi \in L^{2}\left(0, T ; H^{1}(\Omega)\right)$. Now we have identified $J$. Therefore, we want to pass to the limit in

$$
\begin{aligned}
& \int_{\Omega_{T}} J_{\varepsilon} \cdot \eta \\
& =\int_{\Omega_{T}} m_{\varepsilon}\left(u_{\varepsilon}\right) \nabla \\
& \times\left[k \Delta^{2} u_{\varepsilon}\right. \\
& \left.\quad+\left(-a\left(u_{\varepsilon}\right) \Delta u_{\varepsilon}-\frac{a^{\prime}\left(u_{\varepsilon}\right)}{2}\left|\nabla u_{\varepsilon}\right|^{2}+f\left(u_{\varepsilon}\right)\right)\right] \eta,
\end{aligned}
$$

where $\eta \in L^{2}\left(0, T ; H^{1}\left(\Omega, R^{3}\right)\right) \cap L^{\infty}\left(\Omega_{T}, R^{3}\right)$ with $\eta \cdot n=0$ on $\partial \Omega \times(0, T)$ and the left hand side converges to $\int_{\Omega_{T}} J \cdot \eta$. Since $\nabla \Delta^{2} u_{\varepsilon}$ may not have a limit in $L^{2}\left(\Omega_{T}\right)$, we integrate the first term on the right hand side of (50) by parts to get

$$
\begin{aligned}
k \int_{\Omega_{T}} m_{\varepsilon}\left(u_{\varepsilon}\right) \nabla \Delta^{2} u_{\varepsilon} \cdot \eta d x d t \\
\quad=-k \int_{\Omega_{T}} \Delta^{2} u_{\varepsilon} m_{\varepsilon}\left(u_{\varepsilon}\right) \nabla \eta d x d t \\
\quad-k \int_{\Omega_{T}} \Delta^{2} u_{\varepsilon} m_{\varepsilon}^{\prime}(u) \nabla u_{\varepsilon} \cdot \eta d x d t=I+I I .
\end{aligned}
$$

It is easily proved that $m_{\varepsilon}\left(u_{\varepsilon}\right) \rightarrow m(u)$ uniformly. Hence, we have

$$
m_{\varepsilon}\left(u_{\varepsilon}\right) \longrightarrow m(u) \quad \text { a.e. in } \Omega_{T} \text {. }
$$

Since $\Delta^{2} u_{\varepsilon} \rightarrow \Delta^{2} u$ weakly in $L^{2}\left(\Omega_{T}\right)$ and $m_{\varepsilon}\left(u_{\varepsilon}\right)$ is uniformly bounded, we conclude

$$
\begin{array}{r}
k \int_{\Omega_{T}} \Delta^{2} u_{\varepsilon} m_{\varepsilon}\left(u_{\varepsilon}\right) \nabla \eta d x d t \longrightarrow k \int_{\Omega_{T}} \Delta^{2} u(m(u) \nabla \eta) d x d t \\
\text { as } \varepsilon \longrightarrow 0 .
\end{array}
$$

Now we pass to the limit in $I I$. We consider the case $n>1$. As for $m(u)$, we have $m_{\varepsilon}^{\prime}\left(u_{\varepsilon}\right) \rightarrow m^{\prime}(u)$ uniformly, which gives

$$
m_{\varepsilon}^{\prime}\left(u_{\varepsilon}\right) \longrightarrow m^{\prime}(u), \quad \text { a.e. in } \Omega_{T} \text {. }
$$

By using

$$
\nabla u_{\varepsilon} \longrightarrow \nabla u, \quad \text { in } L^{2}\left(\Omega_{T}\right), \quad \text { and a.e. in } \Omega_{T},
$$

and the fact that $m_{\varepsilon}^{\prime}\left(u_{\varepsilon}\right)$ is uniformly bounded, a generalized version of the Lebesgue convergence theorem yields

$$
m_{\varepsilon}^{\prime}\left(u_{\varepsilon}\right) \nabla u_{\varepsilon} \longrightarrow m^{\prime}(u) \nabla u, \quad \text { in } L^{2}\left(\Omega_{T}\right) .
$$

Hence

$$
\begin{aligned}
& k \int_{\Omega_{T}} \Delta^{2} u_{\varepsilon} m_{\varepsilon}^{\prime}(u) \nabla u_{\varepsilon} \cdot \eta d x d t \\
& \quad \longrightarrow k \int_{\Omega_{T}} \Delta^{2} u m^{\prime}(u) \nabla u \cdot \eta d x d t,
\end{aligned}
$$

where we use the fact that $\eta \in L^{\infty}\left(\Omega_{T}\right)$.

To complete the proof of Theorem 4 we have to show

$$
\begin{gathered}
\int_{\Omega_{T}} m_{\varepsilon}\left(u_{\varepsilon}\right) \nabla\left(-a\left(u_{\varepsilon}\right) \Delta u_{\varepsilon}-\frac{a^{\prime}\left(u_{\varepsilon}\right)}{2}\left|\nabla u_{\varepsilon}\right|^{2}+f\left(u_{\varepsilon}\right)\right) \\
\cdot \eta d x d t \\
\longrightarrow \int_{\Omega_{T}} m(u) \nabla\left(-a(u) \Delta u-\frac{a^{\prime}(u)}{2}|\nabla u|^{2}+f(u)\right) \\
\cdot \eta d x d t .
\end{gathered}
$$


In fact, we have

$$
\begin{aligned}
\nabla( & \left.-a\left(u_{\varepsilon}\right) \Delta u_{\varepsilon}-\frac{a^{\prime}\left(u_{\varepsilon}\right)}{2}\left|\nabla u_{\varepsilon}\right|^{2}+f\left(u_{\varepsilon}\right)\right) \\
= & -a^{\prime}\left(u_{\varepsilon}\right) \nabla u_{\varepsilon} \Delta u_{\varepsilon}-a\left(u_{\varepsilon}\right) \nabla \Delta u_{\varepsilon}-\frac{a^{\prime \prime}\left(u_{\varepsilon}\right)}{2} \nabla u_{\varepsilon}\left|\nabla u_{\varepsilon}\right|^{2} \\
& -a^{\prime}\left(u_{\varepsilon}\right)\left(\nabla u_{\varepsilon} \cdot \nabla\right) \nabla u_{\varepsilon}+f^{\prime}\left(u_{\varepsilon}\right) .
\end{aligned}
$$

On the other hand, we know

$$
\begin{aligned}
m_{\varepsilon}\left(u_{\varepsilon}\right) & \longrightarrow m(u), \quad \text { uniformly, } \\
\nabla u_{\varepsilon} & \longrightarrow \nabla u \quad \text { strongly in } L^{2}\left(\Omega_{T}\right), \\
\nabla \Delta u_{\varepsilon} & \longrightarrow \nabla \Delta u \quad \text { strongly in } L^{2}\left(\Omega_{T}\right), \\
D^{2} u_{\varepsilon} & \longrightarrow D^{2} u \quad \text { strongly in } L^{2}\left(\Omega_{T}\right) .
\end{aligned}
$$

Hence, when $\varepsilon \rightarrow 0$, the right hand side of the inequality tends to zero. Similarly, we can pass to the limit, and this finishes the proof of Theorem 4 .

\section{Conflict of Interests}

The authors declare that they have no conflict of interests in this paper.

\section{References}

[1] G. Gompper and J. Goos, "Fluctuating interfaces in microemulsion and sponge phases," Physical Review E, vol. 50, no. 2, pp. 1325-1335, 1994.

[2] I. Pawłow and W. M. Zajączkowski, "A sixth order CahnHilliard type equation arising in oil-water-surfactant mixtures," Communications on Pure and Applied Analysis, vol. 10, no. 6, pp. 1823-1847, 2011.

[3] Z. Wang and C. Liu, "Some properties of solutions for the sixth-order Cahn-Hilliard-type equation," Abstract and Applied Analysis, vol. 2012, Article ID 414590, 24 pages, 2012.

[4] C. Liu and Z. Wang, "Optimal control for a sixth order nonlinear parabolic equation," Mathematical Methods in the Applied Sciences, 2013.

[5] G. Schimperna and I. Pawłow, "On a class of Cahn-Hilliard models with nonlinear diffusion," SIAM Journal on Mathematical Analysis, vol. 45, no. 1, pp. 31-63, 2013.

[6] C. Liu, "Regularity of solutions for a sixth order nonlinear parabolic equation in two space dimensions," Annales Polonici Mathematici, vol. 107, no. 3, pp. 271-291, 2013.

[7] J. Simon, "Compact sets in the space $L^{p}(0, T ; B)$, , Annali di Matematica Pura ed Applicata. Serie Quarta, vol. 146, pp. 6596, 1987.

[8] J. L. Lions, Quelques méthodes de résolution des problèmes aux limites nonlinéaires, Dunod, Paris, France, 1969. 


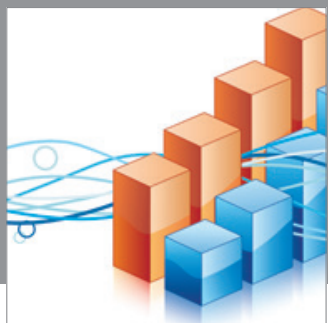

Advances in

Operations Research

mansans

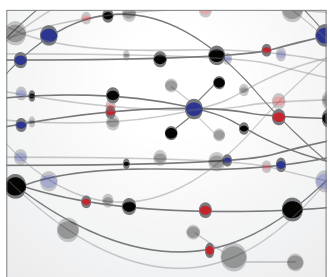

The Scientific World Journal
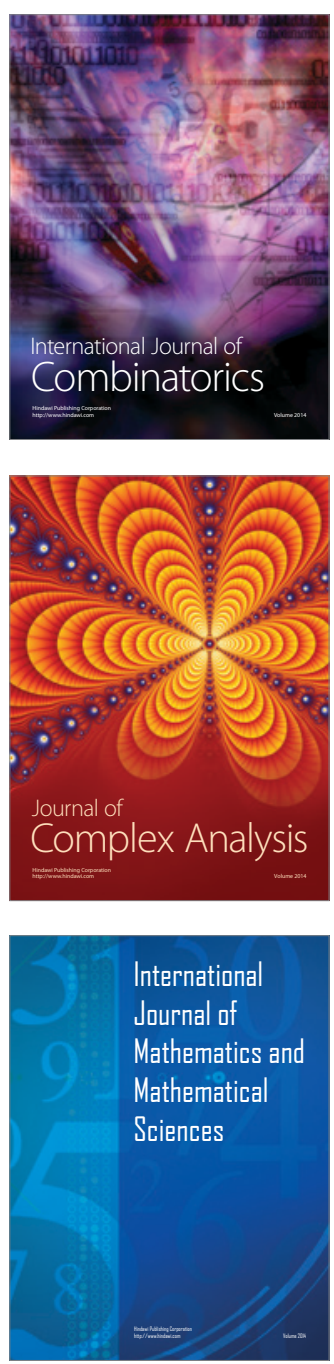
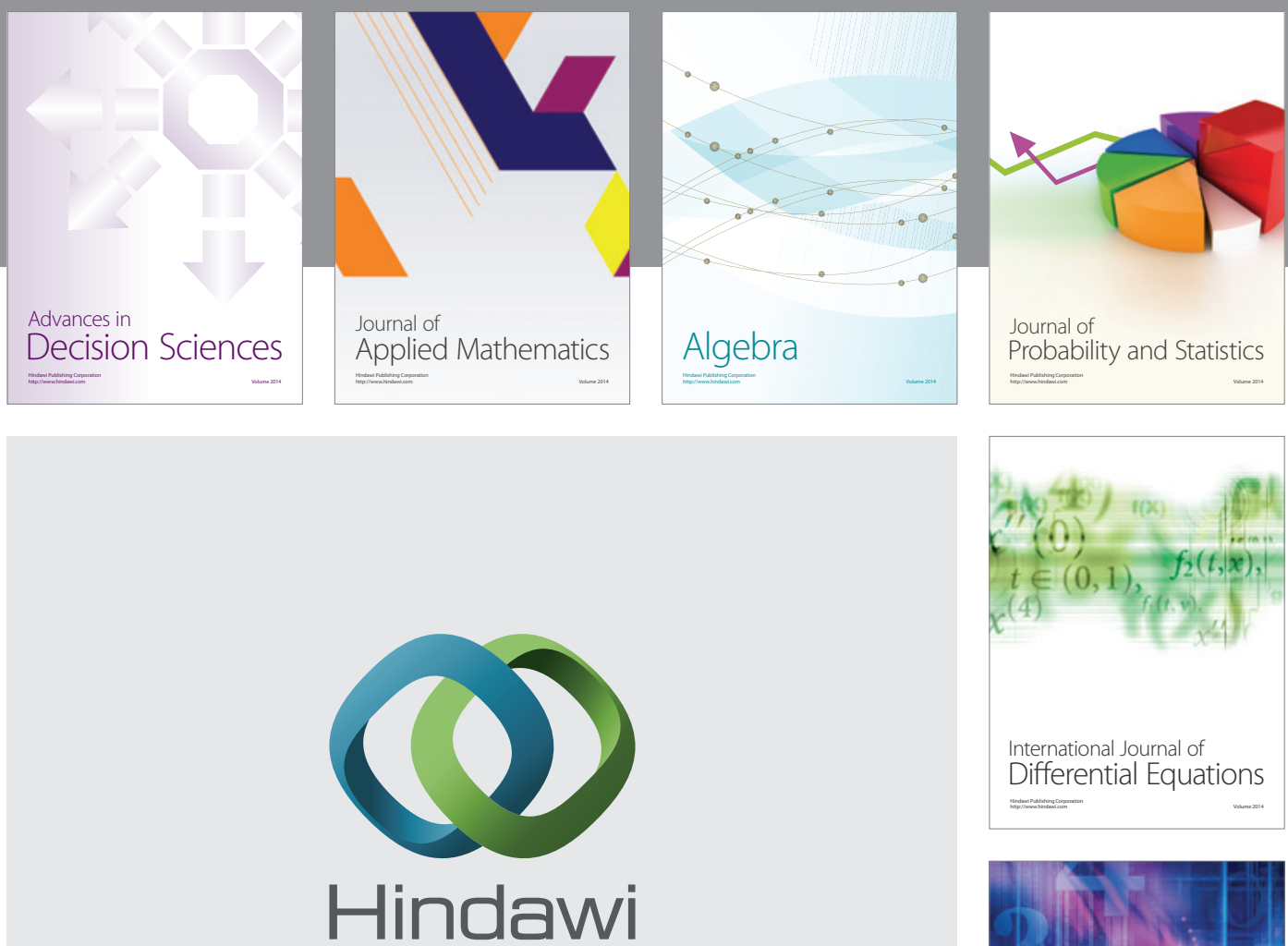

Submit your manuscripts at http://www.hindawi.com
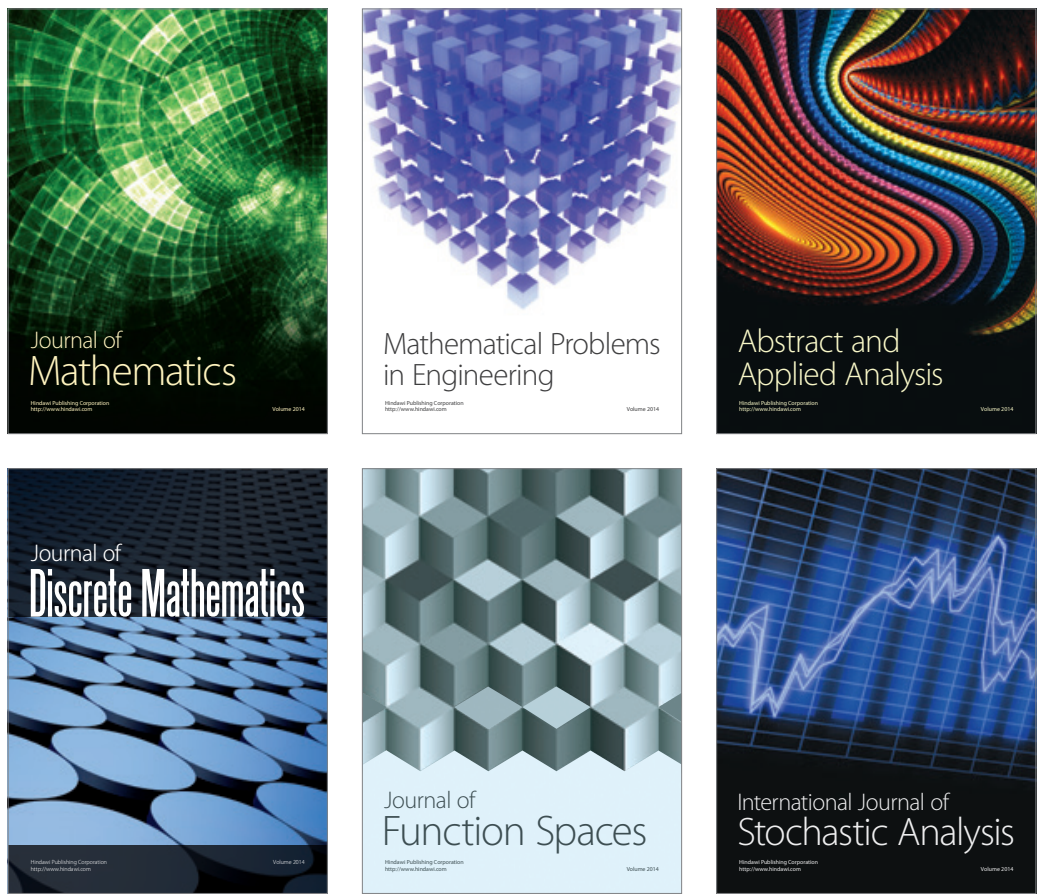

Journal of

Function Spaces

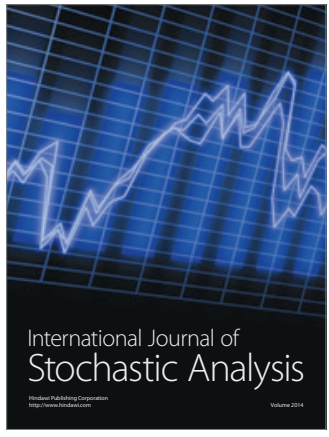

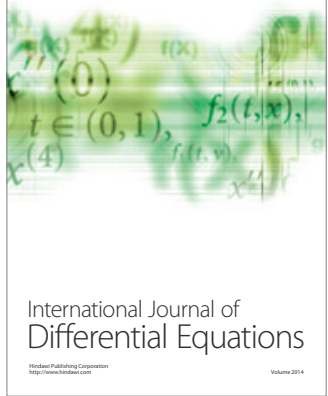
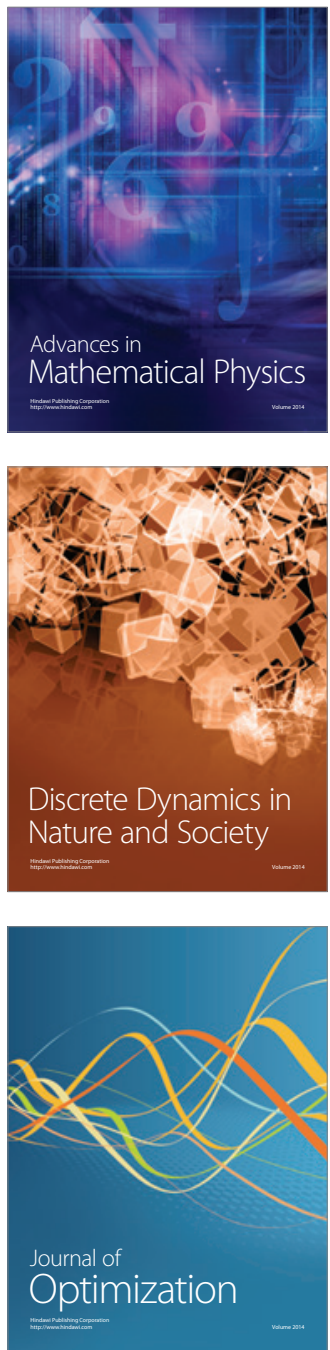